\title{
Fluid-structure interaction modelling of a PWR fuel assembly subjected to axial flow
}

\author{
Guillaume Ricciardi , \\ CEA CADARACHE DEN/DTN/STRI/LHC, 13108, Saint-Paul-Lez-Durance Cedex, France
}

\begin{abstract}
Nuclear industry needs tools to design reactor cores in case of earthquake. A fluid-structure model simulating the response of the core to a seismic excitation have been developed. Full scale tests considering one fuel assembly are performed to identify coefficients (added mass and damping) that will be used as inputs in the models. Tests showed that the axial water flow induced an added stiffness. In the paper, an expression of the model accounting for the fluid in the fuel assembly with a porous media model and in the by-passes with a leakage flow model is developed. Numerical simulations are compared to experiments and showed good agreement.
\end{abstract}

Key words: fuel assembly, modelling, fluid-structure interaction, added stiffness

\section{Introduction}

Earthquakes can irreversibly damage nuclear power plants especially in the core, where the nuclear fuel assemblies containing enriched uranium dioxide have to be particularly resistant. Before building a nuclear power plant, it is necessary to make sure that the core will resist the worst possible earthquake conditions liable to occur at the reactor site. Therefore, safety measures are required to insure the drop of control rods and that the core is cooled when the fuel assembly spacer grids strike each other during seismic excitation of a Pressurized Water Reactors (PWR). A way to insure these two criteria is to prevent the spacer grids from buckling. Engineers need special tools for designing and maintaining reactor cores.

The reactor core made of fuel assemblies is subjected to an axial water flow to cool the reactor. The flow strongly modifies the dynamical behaviour of the fuel assemblies (Collard et al., 2004), therefore the identification of the fluid forces is important to provide a relevant modelling of the fuel assemblies behaviour. The first approximation of the fluid forces is to consider them as added mass and damping (Rigaudeau, 1997; Viallet et al., 2003). A more complex expression of these fluid forces is given by Païdoussis (2003) in which the velocity and the relative direction of the flow with respect to the fuel assembly are accounted for. Ricciardi et al. $(2009 \mathrm{a}, \mathrm{b})$ proposed a porous media approach based on the Païdoussis theory.

In previous study (Ricciardi and Boccaccio, 2012), tests dedicated to the identification of the fluid forces acting on a full scale fuel assembly were performed. These tests highlighted an added stiffness effect under axial flow. This phenomenon was first observed in Ricciardi et al. (2010), and then discussed in Ricciardi

Email address: guillaume.ricciardi@cea.fr (Guillaume Ricciardi). 
and Boccaccio (2012). Identified coefficients of stiffness, damping and mass showed a strong dependence on the lateral by-passes. These by-passes are necessary to allow the displacement of the fuel assemblies. Further tests involving fluid measurements in the by-passes (Ricciardi and Boccaccio, 2014b) showed that fluid velocity fluctuations were induced by the fuel assembly displacement. A delay was observed between the displacement and the fluid fluctuation. The delay decreases with the increase of the axial velocity, thus the phenomenon involved should be related to the fluid convection. A first attempt to model the added stiffness was made by Ricciardi and Boccaccio (2015) based on Bernoulli equation and an artificial delay and not accounting for the by-passes. In this paper a model accounting for the flow in the fuel assembly and in the by-passes is proposed.

\section{Experimental Apparatus}

HERMES T is a single phase hydraulic loop that can handle full scale PWR $1300 \mathrm{MW}$ fuel assemblies. The pump can supply $1200 \mathrm{~m}^{3} / \mathrm{h}$ in axial flow and $400 \mathrm{~m}^{3} / \mathrm{h}$ in cross-flow, at 35 bar and $170^{\circ} \mathrm{C}$. Therefore, the flow rate is similar to the PWR condition, the lower temperature (PWR operates at $315^{0} \mathrm{C}$ ) allows to provide accurate measurement devices to the test-section. In the present study, only axial flow is considered at $50^{\circ} \mathrm{C}$.

The fuel assembly used is made up of 25 guide tubes and 264 fuel rods, each having a height of $4.5 \mathrm{~m}$ and linear density of about $200 \mathrm{~kg} / \mathrm{m}$. Fuel rods contain uranium dioxide pellets and have a diameter of $9.5 \mathrm{~mm}$. The pitch of the fuel bundle is $12.5 \mathrm{~mm}$. The fuel assembly is clamped to the test-section at the top and the bottom. The test-section is about $40 \mathrm{~mm}$ larger than the fuel assembly in the excitation direction and $10 \mathrm{~mm}$ larger in the orthogonal direction. Grids of the fuel assembly are around $200 \mathrm{~mm}$ wide. The displacement of the fifth grid is imposed with a hydraulic jack (Fig. 1). An acrylic window allows making optical fluid measurements. The displacements of the grids are measured with LVDT sensors. The movable portion of each sensor is a stainless steel rod with a diameter of $2.5 \mathrm{~mm}$ and is placed across the right by-pass.

Static and dynamic tests are carried out. For static tests, the axial fluid velocity is measured at three altitudes in the fuel assembly and in the by-passes (Fig. 2), namely, between grids 2 and 3, between grids 4 and 5 and between grids 8 and 9 . For each altitude, measurements are made every $2 \mathrm{~mm}$ along a line in each by-pass, and 6 lines in the fuel assembly, homogeneously distributed (Fig. 3). Static tests are performed with the fuel assembly at rest, and with a $10 \mathrm{~mm}$ imposed displacement of the fifth grid toward right by-pass. For dynamic tests, a swept sine ranging from 0 to $3 \mathrm{~Hz}$ with an amplitude of $6 \mathrm{~mm}$ is imposed. Tests are performed under axial flow for three axial velocities $(1.5 \mathrm{~m} / \mathrm{s}, 3 \mathrm{~m} / \mathrm{s}$ and $5 \mathrm{~m} / \mathrm{s})$. The axial component of fluid velocity is measured at three altitudes in the right by-pass (Fig. 1), between grids 2 and 3, between grids 4 and 5 and between grids 8 and 9. In spite of the disturbance of the LVDT sensors, measurements are made in the right by-pass because the fastener device that link the hydraulic jack to the grid is more perturbing the flow in the left by-pass than the LVDT sensors in the right by-pass. The LDV device (TSI TRx60 probe) allows measuring the fluid velocity at only one point, so the same grid excitation is repeated for each measurement location. LDV device measures the velocity of one particle when it meets the measurement volume which is about $1 \mathrm{~mm}^{3}$, thus it is a random process and data are not regularly sampled. The sample rate is about $2.5 \mathrm{kHz}$. To perform spectral analysis, data are resampled by an interpolation method for 2 $\mathrm{kHz}$. For static tests, the mean velocity is calculated from 1024 samples.

For confidential reason, all the velocity values are given dimensionless and normalized by the same quantity.

\section{Coupled fluid-structure modelling}

The modelling proposed by Ricciardi et al. $(2009 \mathrm{a}, \mathrm{b})$ is based on a porous medium approach. This approach gives access to an equivalent fluid model and an equivalent structure model both defined on the whole domain. Motion equations for the equivalent fluid and the equivalent structure are first established separately. For the fluid part, global fluid flow equations through the rod bundle are obtained by local spatially averaging the Navier Stokes equations written with an Arbitrary Lagragian Eulerian approach. The resulting equivalent fluid is characterized by an equivalent velocity and an equivalent pressure both defined in the whole domain. 
Structure related effects on fluid are accounted for by a body force also defined in the whole domain. The structure equations are also space averaged but fuel assembly by fuel assembly, each fuel assembly is modelled as an equivalent structure satisfying a Timoshenko beam model with a nonlinear behaviour. For each fuel assembly, the unknowns are reduced to the displacement of the mean line $u$ and the rotation of the cross section $\theta$, such as the displacement $U$ of the structure can be obtained by the relation :

$$
\mathbf{U}(x, y)=u(x) \mathbf{e}_{\mathbf{y}}+y \theta(x) \mathbf{e}_{\mathbf{x}}
$$

where $\mathbf{e}_{\mathbf{x}}$ and $\mathbf{e}_{\mathbf{y}}$ are the unity vectors in the $x$ and $y$ directions (Fig. 4).

Fluid related effects on structure are accounted for by a body force which is defined in the whole domain. Finally, fluid related effects on structure and structure related effects on fluid are of opposite sign and are built from expression of fluid forces acting on a rod subjected to an axial flow proposed by Païdoussis (2003).

The equations of the structure motion are :

$$
\begin{aligned}
m_{f a} \frac{\partial^{2} u}{\partial t^{2}} & =\frac{\partial T}{\partial x}+N_{0} \frac{\partial^{2} u}{\partial x^{2}}+\frac{S_{e q}}{d_{g}^{2}}\left(F_{I}+F_{N}+F_{D}-x F_{L} \frac{\partial^{2} u}{\partial x^{2}}\right)-\phi_{s} w_{f a}\left(P_{r}-P_{l}\right), \\
I_{f a} \frac{\partial^{2} \theta}{\partial t^{2}} & =\frac{\partial M}{\partial x}+T
\end{aligned}
$$

where $m_{f a}$ and $I_{f a}$ are the mass and inertial moment per unit length of a fuel assembly, $N_{0}$ is the tension force at the bottom of the fuel assembly, $d_{g}$ is the distance between two fuel rods centre, $S_{e q}$ is cross-section area of the equivalent beam, $T$ is the shear force and $M$ is the bending moment.

The constitutive laws of the fuel assemblies are given by :

$$
\begin{aligned}
& T=G S_{e q}\left(\frac{\partial u}{\partial x}-\theta\right)+\mu_{G} S_{e q} \frac{\partial}{\partial t}\left(\frac{\partial u}{\partial x}-\theta\right), \\
& M=E I_{e q} \frac{\partial \theta}{\partial x}+\mu_{E} I_{e q} \frac{\partial^{2} \theta}{\partial t \partial x}+M_{f r e t},
\end{aligned}
$$

where $G$ is the shear modulus, $E$ is the Young's modulus, $I_{e q}$ is the quadratic moment of a fuel assembly and $\mu_{G}$ and $\mu_{E}$ are structural damping coefficients.

$M_{\text {fret }}$ accounts for a progressive fretting. When the relative rotation is small, the structure has a linear behaviour. When the relative rotation is higher than the friction angle $\theta_{f}$, the fuel rods at the extremities start to slid into the grids. As the relative rotation increases the number of rods sliding increases. This phenomenon is accounted for by the following equations :

$$
\begin{gathered}
M_{\text {fret }}=M_{0}+ \begin{cases}\frac{N_{p}^{4} K_{c} d_{g}^{2}}{12}\left(\theta-\theta_{0}\right) & \text { if }\left(\theta-\theta_{0}\right)<\theta_{f} \\
\frac{N_{p}^{3} F_{r i} d_{g}}{4}-\frac{2 N_{p} F_{r i}^{3}}{3 d_{g} K_{c}^{2}} \frac{1}{\left(\theta-\theta_{0}\right)^{2}} & \text { if }\left(\theta-\theta_{0}\right)>\theta_{f}\end{cases} \\
\theta_{f}=\frac{2 F_{r i}}{N_{p} d_{g} K_{c}},
\end{gathered}
$$

where $M_{0}$ and $\theta_{0}$ are the moment value and the angle position when the fuel assembly changes its displacement direction, $N_{p}$ is the number of fuel rods in one direction, $K_{c}$ is the axial stiffness of a fuel rod and $F_{r i}$ is the friction force between one fuel rod and one grid.

The equations of the equivalent fluid in the fuel assembly are :

$$
\begin{aligned}
& \frac{\partial V_{f a}}{\partial t}+V_{f a} \frac{\partial V_{f a}}{\partial x}=-\frac{1}{\rho_{e q}} \frac{\partial P_{f a}}{\partial x}-\frac{1}{d_{g}^{2} \rho_{e q}} F_{L}, \\
& \frac{\partial V_{c f}}{\partial t}+V_{f a} \frac{\partial V_{c f}}{\partial x}=-\frac{1}{\rho_{e q}}\left(P_{r}-P_{l}\right)-V_{f a} \frac{\partial^{2} u}{\partial t \partial x}-\frac{1}{d_{g}^{2} \rho_{e q}}\left(F_{I}+F_{N}+F_{D}-F_{L} \frac{\partial u}{\partial x}\right),
\end{aligned}
$$


where $V_{f a}$ is the axial component of the mean velocity in the fuel assembly, $V_{c f}$ is the transvers component of the mean velocity in the fuel assembly, $P_{f a}$ is the mean pressure in the fuel assembly, $P_{r}$ is the mean pressure in the right by-pass, $P_{l}$ is the mean pressure in the left by-pass and $\rho_{e q}$ is the equivalent fluid density defined by $\rho_{e q}=\phi \rho$ where $\phi$ is the porosity and $\rho$ is the fluid density.

The fluid forces account for added mass effect $F_{I}$, flow induced damping $F_{N}$, damping in still water $F_{D}$ and axial drag force $F_{L}$ :

$$
\begin{aligned}
& F_{I}=-m_{f}\left(\frac{\partial^{2} u}{\partial t^{2}}-\frac{\partial V_{c f}}{\partial t^{2}}+V_{f a}^{2} \frac{\partial^{2} u}{\partial x^{2}}+2 V_{f a} \frac{\partial^{2} u}{\partial t \partial x}-2 V_{f a} \frac{\partial V_{c f}}{\partial x}\right), \\
& F_{N}=-\frac{1}{2} \rho d_{c} V_{f a} C_{N}\left(\frac{\partial u}{\partial t}-V_{c f}+V_{f a} \frac{\partial u}{\partial x}\right), \\
& F_{D}=-C_{D}\left(\frac{\partial u}{\partial t}-V_{c f}\right), \\
& F_{L}=\frac{1}{2} \rho d_{c} C_{T} V_{f a}^{2},
\end{aligned}
$$

where $m_{f}$ is a virtual mass per unit length, $d_{c}$ is the fuel rod diameter, $\rho$ is the fluid density, $C_{N}, C_{T}$ are drag coefficients and $C_{D}$ is a damping coefficient.

\section{By-pass flow modelling}

Considering the dimensions of the by-passes a one degree of freedom leakage flow model is applied. As in Inada and Hayama (1990a), Inada and Hayama (1990b), Porcher and de Langre (1997) or X. Wu and S. Kaneko (2005) (see also Païdoussis (2003)), the axial component of the fluid dynamic equation is integrated over the width of the by-passes noted $r$ and $l$ respectively for right and left by-pass (Fig. 4):

$$
\int_{h_{l / r}}\left(\frac{\partial v_{x}}{\partial t}+v_{x} \frac{\partial v_{x}}{\partial x}\right) d y=-\int_{h_{l / r}}\left(\frac{1}{\rho} \frac{\partial p}{\partial x}+\nu\left(\frac{\partial^{2} v_{x}}{\partial x^{2}}+\frac{\partial^{2} v_{x}}{\partial y^{2}}\right)\right) d y
$$

where $v_{x}$ is the axial component of the fluid velocity, $p$ is the pressure, $\nu$ is the fluid viscosity and $h_{r}$ and $h_{l}$ are respectively the width of the right and left by-passes.

The mean values of velocities and pressures are defined as follows :

$$
\begin{aligned}
& V_{l}=\frac{1}{h_{l}} \int_{h_{l}} v_{x} d y, \\
& V_{r}=\frac{1}{h_{r}} \int_{h_{r}} v_{x} d y, \\
& P_{l}=\frac{1}{h_{l}} \int_{h_{l}} p d y, \\
& P_{r}=\frac{1}{h_{r}} \int_{h_{r}} p d y .
\end{aligned}
$$

The widths depend on the structure displacement :

$$
\begin{aligned}
& h_{l}=h_{0}+u, \\
& h_{r}=h_{0}-u,
\end{aligned}
$$

where $h_{0}$ is the width of the by-passes when the fuel assembly is in the middle of the test section. 
The viscous effect can be modelled as a term opposed to the flow proportional to the square of the mean velocity :

$$
\int_{h_{l / r}} \nu\left(\frac{\partial^{2} v_{x}}{\partial x^{2}}+\frac{\partial^{2} v_{x}}{\partial y^{2}}\right) d y=C_{h} V_{l / r}^{2},
$$

where $C_{h}$ is a dimensionless friction coefficient.

Replacing Eq.22 in Eq.15, simplifying the convective term and considering the mean values of pressures and velocities, on can obtain the governing equation of the flow in the by-passes :

$$
\begin{gathered}
\frac{\partial V_{l}}{\partial t}+V_{l} \frac{\partial V_{l}}{\partial x}=-\frac{1}{\rho} \frac{\partial P_{l}}{\partial x}+\frac{C_{h}}{\left(h_{0}+u\right)} V_{l}^{2}, \\
\frac{\partial V_{r}}{\partial t}+V_{r} \frac{\partial V_{r}}{\partial x}=-\frac{1}{\rho} \frac{\partial P_{r}}{\partial x}+\frac{C_{h}}{\left(h_{0}-u\right)} V_{r}^{2} .
\end{gathered}
$$

Considering a control volume accounting for the whole width of the test section, the continuity equation for an incompressible fluid gives :

$$
\left(h_{0}+u\right) V_{l}+\left(h_{0}-u\right) V_{r}+\phi w_{f a} V_{f a}=\phi w_{f a} V_{b},
$$

where $V_{b}$ is the bulk velocity imposed at the inlet of the test section.

Considering the continuity equation in both by-passes and taking the difference gives :

$$
\left(h_{0}+u\right) \frac{\partial V_{l}}{\partial x}-\left(h_{0}-u\right) \frac{\partial V_{r}}{\partial x}+2\left(\phi V_{c f}-\phi_{s} \frac{\partial u}{\partial t}\right)=0 .
$$

\section{Comparison experiments simulations}

\subsection{Static case}

Figure 5 shows the axial component of the mean velocity along the by-pass for three axial velocity. One can observe that the simulations reproduce the increase of velocity along the by-pass. At the inlet the bypasses are not alimented and the flow progressively goes from the fuel assembly to the by-passes since the hydraulic diameter of the fuel assembly is smaller than the one of the by-passes. Thus the axial velocity decreases along the fuel assembly, this behaviour is reproduced by the simulation (Fig. 6).

When a displacement is imposed at the fifth grid, the left and right by-passes have different hydraulic diameters resulting in a decrease of the velocity in the by-pass getting narrower and an increase in the by-pass getting wider (Fig. 7). This difference of velocity in the by-passes is reproduced by the simulation although underestimated in the top region of the fuel assembly.

According to the continuity equation (26) the difference of velocity in the by-pass induces a transverse flow in the fuel assembly (Fig. 8). This flow result in a force that tends to bring the fuel assembly in its equilibrium position, thus this phenomenon can be interpreted as an added stiffness effect. One can observe that the hysteresis and the increase of stiffness under axial flow are reproduced by the model (Fig. 9).

\subsection{Dynamic case}

The fuel assembly motion induces fluid fluctuations in the by-passes, these fluctuations show different patterns considering the excitation frequency, the axial velocity and the altitude. Fluctuations between the grids 2 and 3 are small, simulations seem to underestimate them (Fig. 10) nevertheless, experiments show structure induced fluctuations barely higher than turbulent fluctuations. Simulations reproduce the frequency dependency of fluctuations (Figs. 10, 11 and 12), but fluctuations are underestimated especially between the grids 8 and 9 which is coherent with the static case results (Fig. 7). 
Figure 13 shows the phase between the displacement of the fuel assembly and the fluctuations in the right by-pass. One can observe that the simulations reproduce the general increase of the phase with the excitation frequency with a very good matching for an axial velocity of $1.5 \mathrm{~m} / \mathrm{s}$. Assuming a linear increase of the phase, it can be related to a delay (Ricciardi and Boccaccio, 2014b). Simulations reproduce the decrease of the delay with the axial velocity. Although there are some discrepancies, simulations agree with the experiment on the fact that the delay related to grids 2-3 and grids 4-5 are close and smaller than those related to grids $8-9$.

Ricciardi and Boccaccio (2014a) has established an identification method based on a proper orthogonal decomposition (Clement et al., 2014) giving mass stiffness and damping coefficient per unit of length considering the structure shape at resonance. This method is applied on simulations and experiments. For confidential reasons all mass, damping and stiffness parameters are given dimensionless based on characteristic length, mass, quadratic momentum, Young's modulus and frequency of a fuel assembly. Simulations agree with experiments on the increase of stiffness (Fig. 15) and damping (Fig. 16) with the axial velocity. One can observe some differences between experiments and simulations for the mass (Fig. 17) and the frequency (Fig. 18). This difference can be due to the simplified Timoshenko beam model of the structure which show difference in the mode shape at resonance (Fig. 19). Nevertheless one can observe that the influence of the axial flow on the structure shape at resonance is reproduced.

\section{Discussion}

The model proposed reproduces the added stiffness phenomenon experimentally observed, moreover it gives an explanation : the added stiffness is due to the transvers flow induced by the difference of fluid velocity in the two by-passes (Fig. 8). One could wonder how can the model reproduce this phenomenon whereas it fails to reproduce the difference of fluid velocity between the grids 8 an 9 (Fig. 7). One raison could be that at that level the wrong evaluation of the fluid force does not have important consequences on the structure behaviour since it is close to the clamped boundary condition. One could also wonder why the model fails to reproduce the velocity at that level. This could be due to the singularity of the top nozzle which is not accounted for in the model. Moreover, the dimensionless friction coefficient $C_{h}$ is taken constant whereas it is known to depend on the Reynolds number, and the Reynolds appears to take different values along the by-passes.

The singularities of the grids are not accounted for, their effects are modelled globally by the space averaging operation of the porous media modelling. This is a simplification and this singularities may have an effect on the global behaviour. Figures 10, 11 and 12 show some differences in fluid fluctuations amplitudes between experiments and simulations. The singularity may explain a part of this difference, but one has remind that local measured values showing some spatial variation (Fig. 3) are compared to mean calculated quantities.

It is interesting to observe that according to the model the same coefficient, $C_{N}$ and $C_{D}$ are responsible for the added stiffness and damping, and that although the added mass is modelled nondependent on the axial velocity the identification gives a mass increasing with the axial velocity. One has to related this with the delay between the structure displacement and the fluid fluctuations observed experimentally and by the simulations. The fluid forces induced by the by-passes result in an added force in static case but as the delay become significant compared to the excitation frequency the added stiffness decreases since it is no more in phase with the structure displacement. Thus in the linear identification process the evolution of added stiffness with the frequency is compensated by an increase of the masse parameter since the increase of resonance frequency is moderate (Fig. 18).

The model and experimental results presented involve one fuel assembly with large by-passes, in a PWR core by-passes and the distance between two fuel assemblies are smaller than the by-passes of the experiments, thus one may presume that added stiffness effect would be less important in a PWR. Nevertheless, the dynamic of a whole core is more complex because the fluid velocities on each side of a fuel assembly will depend on the displacement of the neighbouring fuel assemblies and the added stiffness effect observed in the experimental case would result in a coupling force between fuel assemblies. 


\section{Conclusion}

A fluid structure model simulating the behaviour of a fuel assembly was proposed. The model account for the fluid in the fuel assembly with a porous media model and in the by-passes with a leakage flow model. The simulations reproduced the added stiffness observed experimentally, comparison of the fluid fluctuation in the by-passes showed good agreement, although some discrepancies where observed. One way to improve the accuracy of the model could be to take into account the grids singularities. The added stiffness is due to the difference of fluid velocity between the by-passes induced by the displacement of the fuel assembly, this difference induces a transverse flow responsible for the added stiffness. One question remain, what are the consequences of this added stiffness phenomenon on whole reactor core with several fuel assemblies. This question will be assessed in further study.

\section{Acknowledgements}

The authors are grateful for the financial support of the Electicité de France (EDF) and AREVA.

\section{References}

Collard, B., Pisapia, S., Broc, D., Bellizzi, S., 2004. Flow induced damping of a PWR fuel assembly. Proccedings of the 8th International Conference on Flow-Induced Vibration - FIV 2004, Paris, France, 6-9 July 2004, pp. 307-312.

Clement, S., Bellizzi, S., Cochelin, B. and Ricciardi, G., 2014. Sliding Window Proper Orthogonal Decomposition : application to linear and nonlinear modal identification. Journal of Sound and Vibration. Volume 333, Issue 21, 13 October 2014, p. 5312-5323.

Inada, F. and Hayama, S., 1990. A study on leakage-flow-induced vibrations. Part I: Fluid-dynamic forces and moments acting on the walls of a narrow tapered passage. Journal of Fluids and Structures, Volume 4, Issue 4, July 1990, p. 395-412

Inada, F. and Hayama, S., 1990. A study on leakage-flow-induced vibrations. Part II: Stability analysis and experiments for two-degree-of-freedom systems combining translational and rotational motions. Journal of Fluids and Structures, Volume 4, Issue 4, July 1990, p. 413-428

Païdoussis, M.P., 2003. Fluid-Structure Interactions : Slender Structures and Axial Flow, Vol 2. Elsevier Academic Press, London.

Pisapia, S., Collard, B., Bellizzi, S., Mori, V., 2003. Modal testing and identification of a PWR fuel assembly. In: Vejvoda, S. (Ed.), Transactions of the 17th International Conference on Structural Mechanics in Reactor Technology (SMIRT 17), Prague, Czech Republic, Paper C01-4.

Porcher, G., and de Langre, E., 1997. A friction-based model for fluidlastic forces induced by axial flow. Proceedings 4th International Symposium on Fluid-Structure Interactions, Aeroelasticity, Flow-Induced Vibration and Noise (eds M.P. Païdoussis et al.), Vol. II, AD-Vol. 53-2, pp.67-74 New York : ASME.

Ricciardi, G., Bellizzi, S., Collard, B., Cochelin, B., 2009. Modelling Pressurized Water Reactor cores in terms of porous media. Journal of Fluids and Structures, Vol. 25, 2009, p. 112-133

Ricciardi, G., Bellizzi, S., Collard, B., Cochelin, B., 2009. Row of fuel assemblies analysis under seismic loading: Modelling and experimental validation. Nuclear Engineering and Design, Vol. 239, 2009, p. 26922704

Ricciardi, G., Bellizzi, S., Collard, B., Cochelin, B., 2010. Fluid-structure interaction in a 3 by 3 reduced scale fuel assembly. Science and Technology of Nuclear Installations Volume 2010 (2010), Article ID 517471, 8 pages doi:10.1155/2010/51747.

Ricciardi, G., Boccaccio, E., 2012. stiffening Of A Fuel Assembly Under Axial Flow. Proccedings of the 10th International Conference on Flow-Induced Vibration (\& Flow-Induced Noise)- FIV2012, Dublin, Ireland, 2-6 july 2012, pp. 391-397. 
Ricciardi, G., Boccaccio, E., 2014. Mass, stiffness and damping identification for a PWR fuel assembly by a POD method. Journal of Pressure Vessel Technology. 2014; 136(6):061303-061303-9. doi: $10.1115 / 1.4028559$

Ricciardi, G., Boccaccio, E., 2014. Measurements of fluid fluctuations around an oscillating nuclear fuel assembly. Journal of Fluids and Structures. Volume 48, July 2014, Pages 332-346.

Ricciardi, G., Boccaccio, E., 2015. Modelling of the Flow Induced Stiffness of a PWR Fuel Assembly. Nuclear Engineering and Design, 2015, vol 282, p. 8-14.

Rigaudeau, J., 1997. Grid modelling and strength criterion in the lateral response of PWR fuel assemblies under accident conditions. $5^{\text {th }}$ International Conference on Nuclear Engineering Nice, France, ICONE52568 .

Viallet, E., Bolsee, G., Ladouceur, B., Goubin, T., Rigaudeau, J., 2003. Validation of PWR core seismic models with shaking table tests on interacting scale 1 fuel assemblies. Transactions of the 17th International Conference on Structural Mechanics in Reactor Technology (SMIRT 17) Prague, Czech Republic.

$\mathrm{Wu}, \mathrm{X}$. and Kaneko, S., 2005. Linear and nonlinear analyses of sheet flutter induced by leakage flow. Journal of Fluids and Structures, Volume 20, Issue 7, October 2005, p. 927-948. 


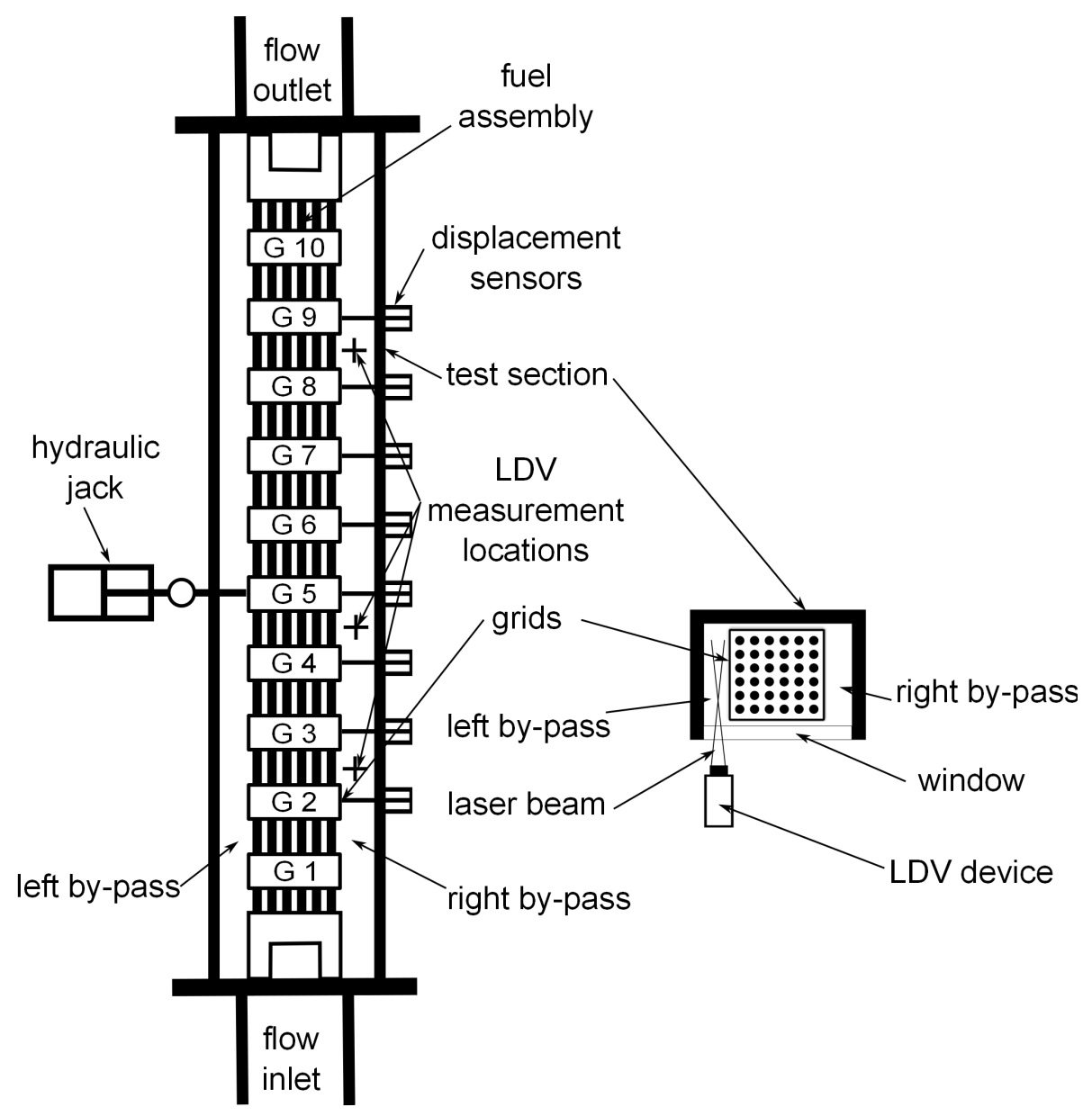

Fig. 1. Experimental apparatus.

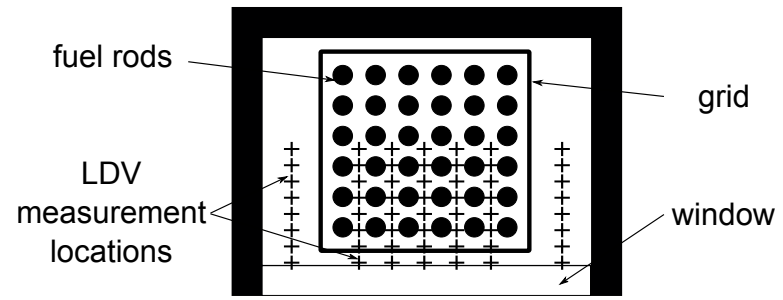

Fig. 2. Indicative diagram of measurement locations for static test in the fuel assembly. 
Dimensionless velocity

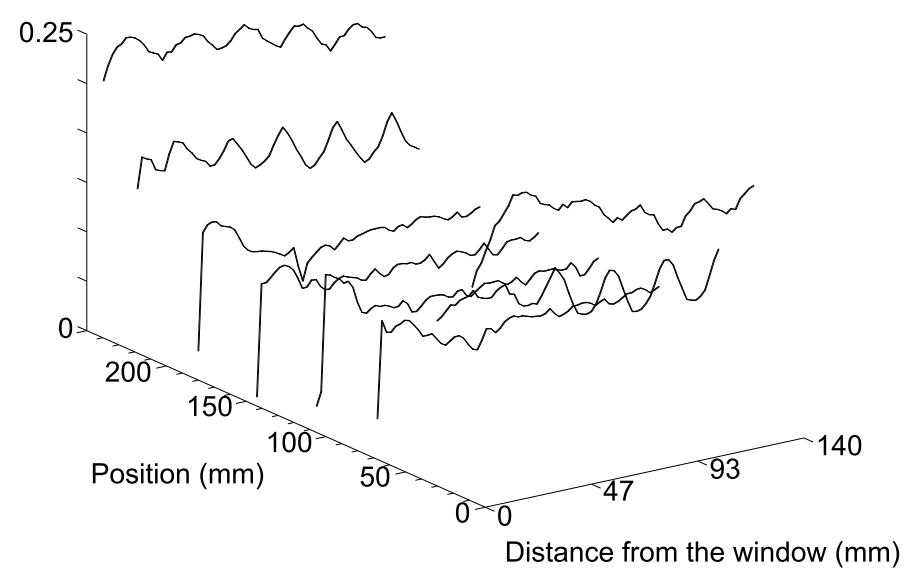

Fig. 3. LDV measurements in the fuel assembly and the by-passes between the grids 8 and 9 at $1.5 \mathrm{~m} / \mathrm{s}$ axial velocity.

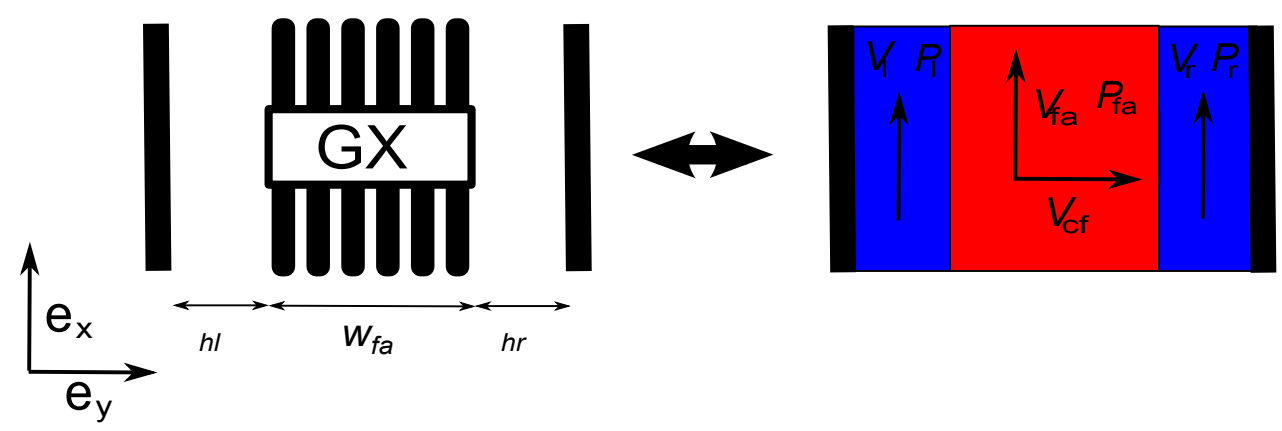

Fig. 4. Modelling of the flow in the fuel assembly and the by-passes. 


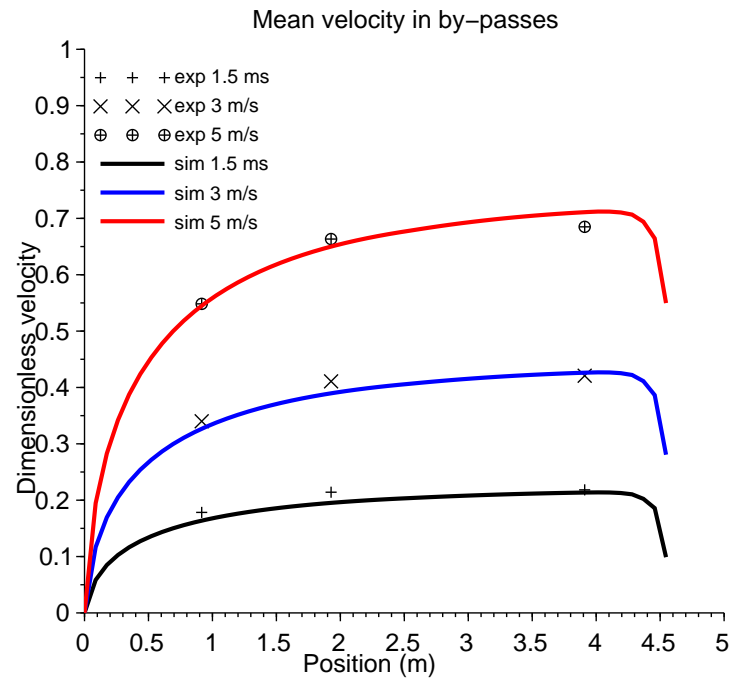

Fig. 5. Axial component of the mean velocity in the by-pass for various axial velocities for the fuel assembly is in the middle of the test section.

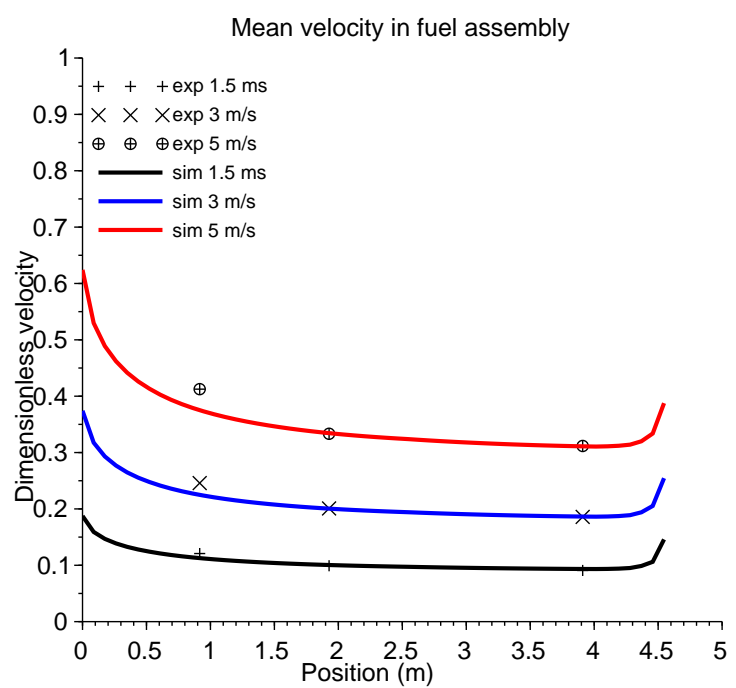

Fig. 6. Axial component of the mean velocity in the fuel assembly for various axial velocities when the fuel assembly is in the middle of the test section. 


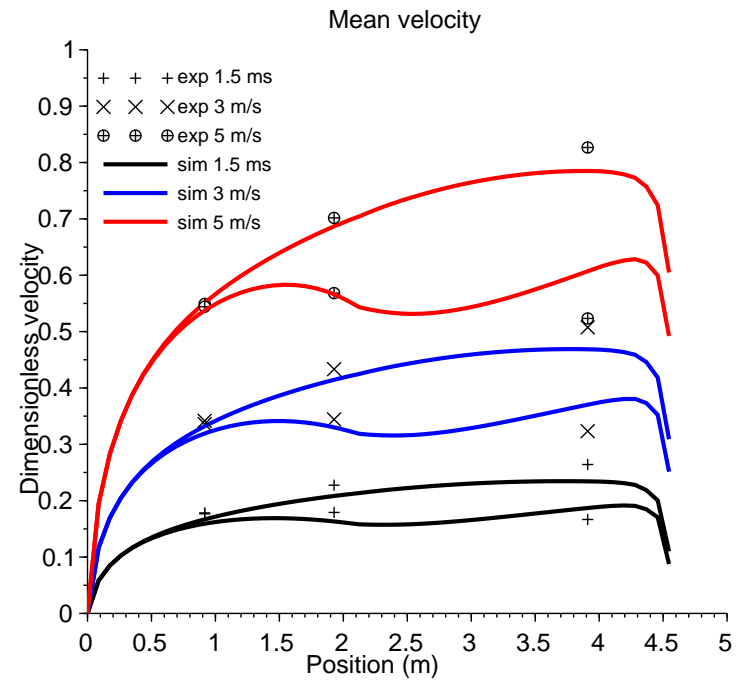

Fig. 7. Axial component of the mean velocity in the by-pass for various axial velocities for a $10 \mathrm{~mm}$ displacement of the fuel assembly fifth grid.

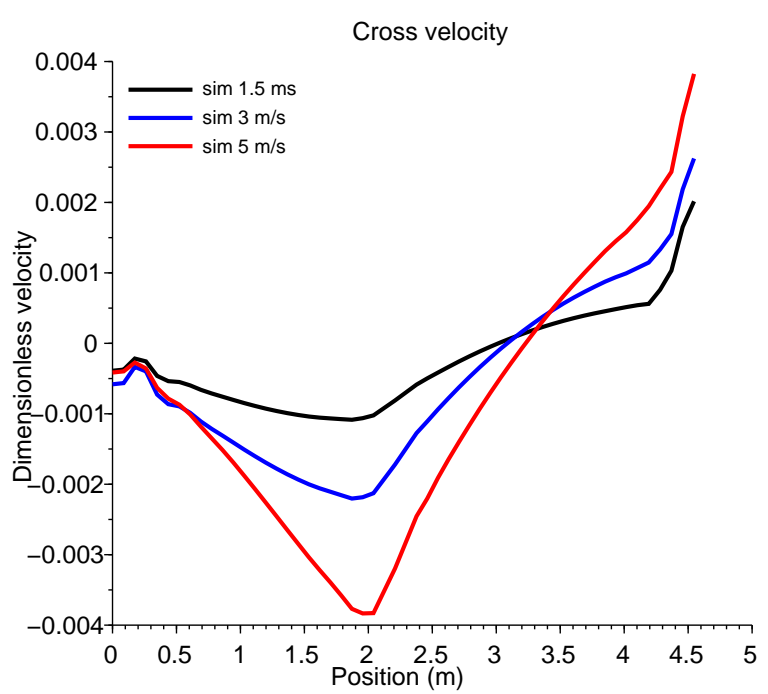

Fig. 8. Transverse component of the mean velocity in the fuel assembly for various axial velocities for a $10 \mathrm{~mm}$ displacement of the fuel assembly fifth grid. 


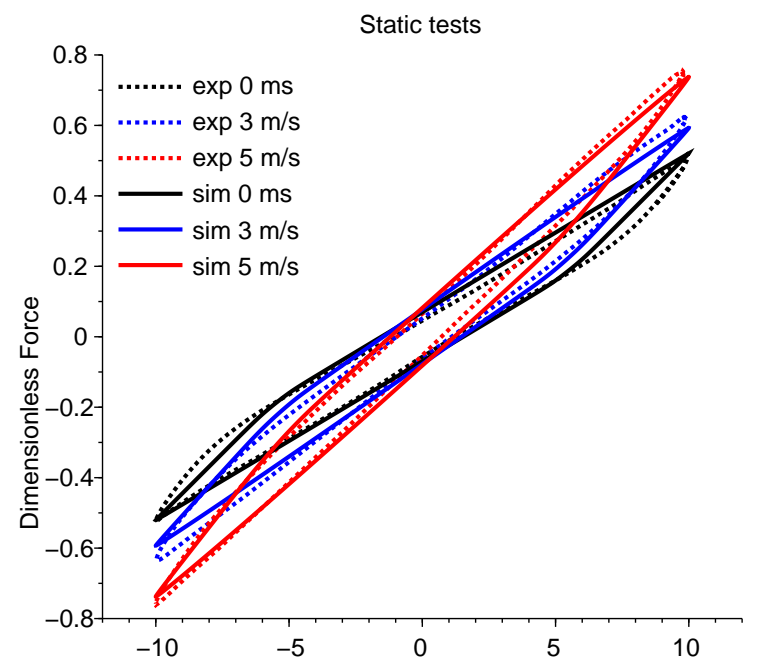

Fig. 9. Force applied byieplaqeyarntumm) 


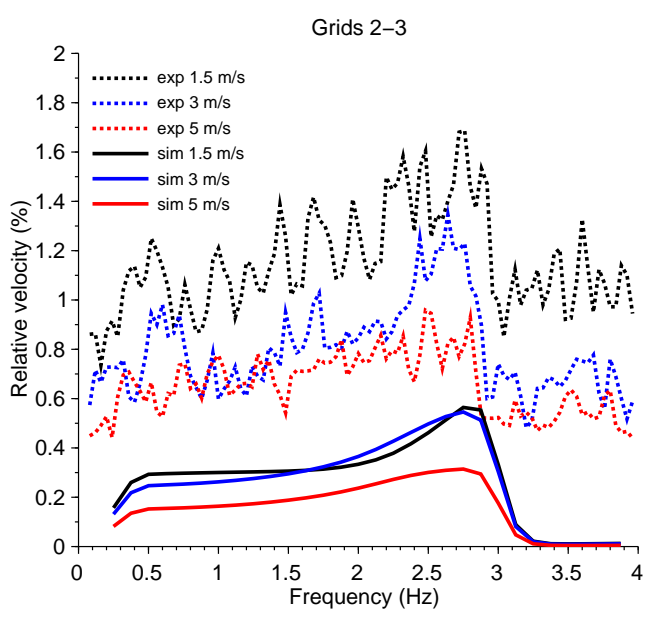

Fig. 10. PSD of the relative axial velocity in the right by-pass between grids 2 and 3 for various axial velocities, in dynamic tests case.

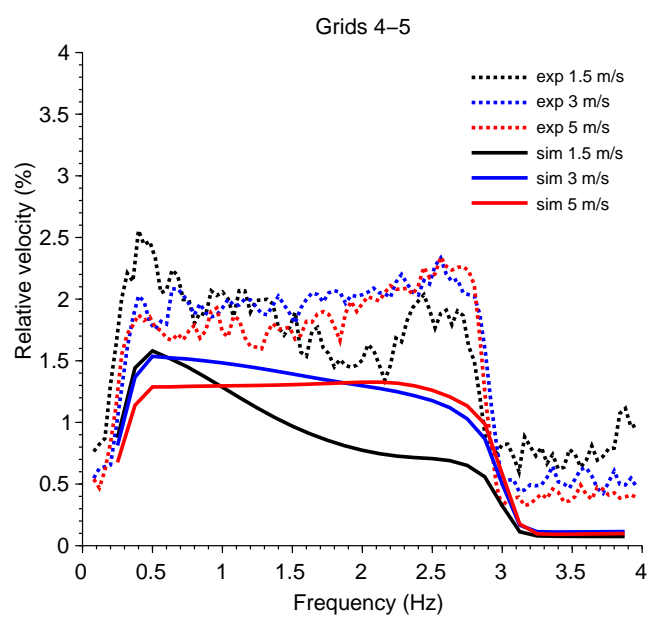

Fig. 11. PSD of the relative axial velocity in the right by-pass between grids 4 and 5 for various axial velocities, in dynamic tests case. 


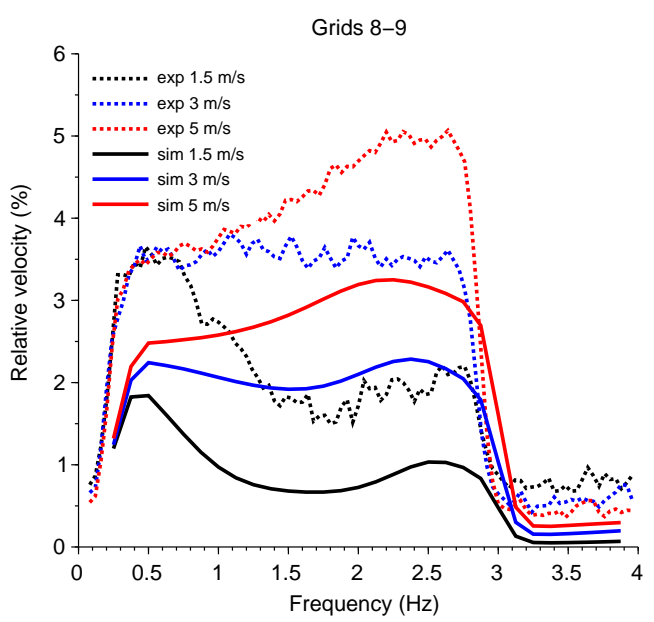

Fig. 12. PSD of the relative axial velocity in the right by-pass between grids 8 and 9 for various axial velocities, in dynamic tests case.

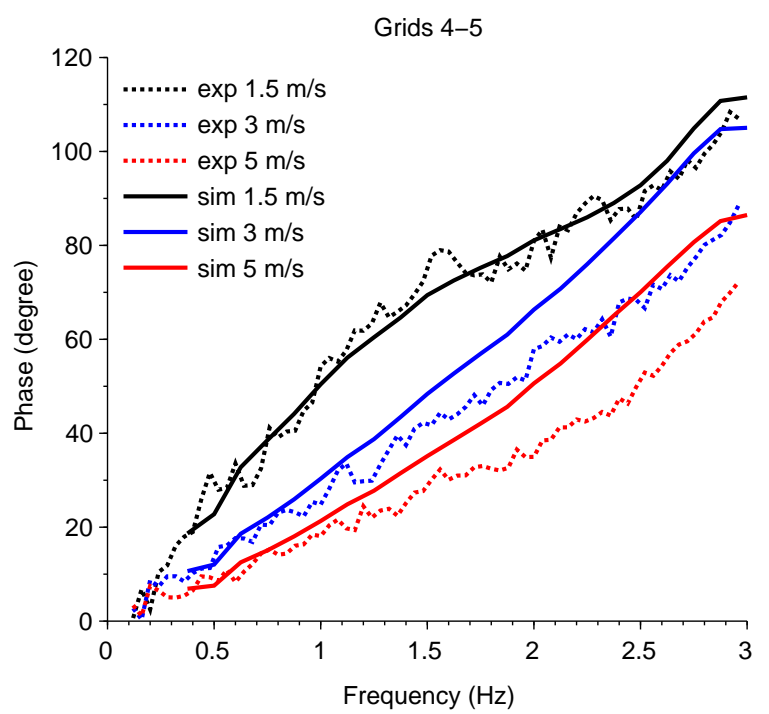

Fig. 13. Phase between the imposed displacement and the fluid velocity in the right by-pass for various axial velocities between grids 4 and 5. 


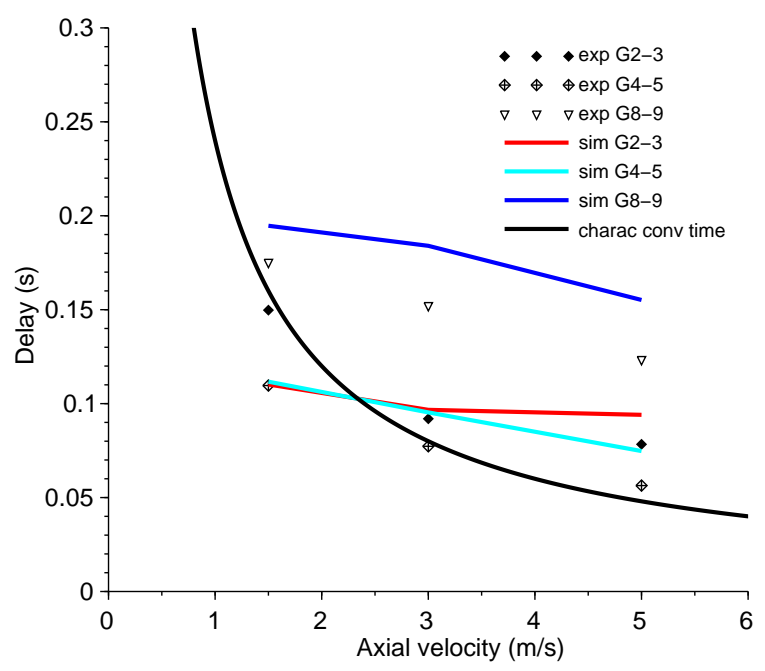

Fig. 14. Time delay between the imposed displacement and the fluid velocity in the right by-pass.

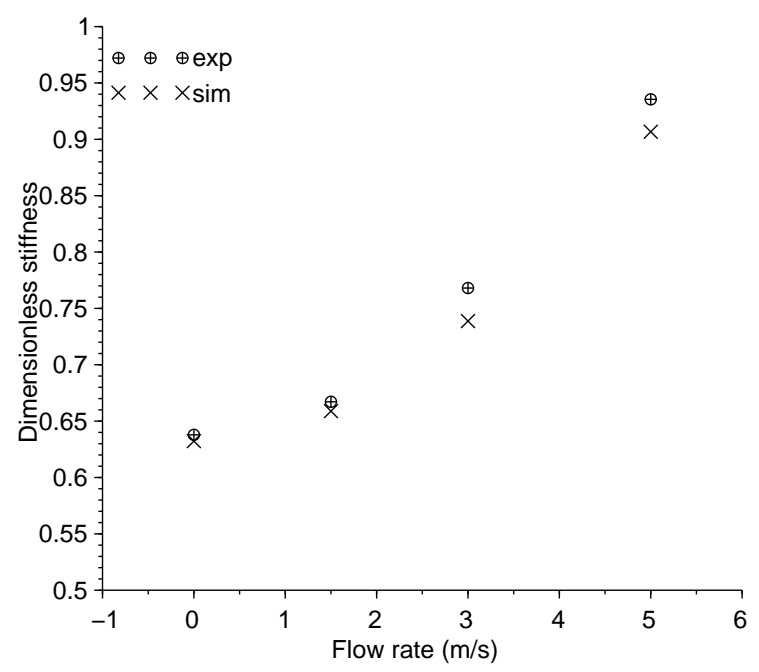

Fig. 15. Stiffness parameter. 


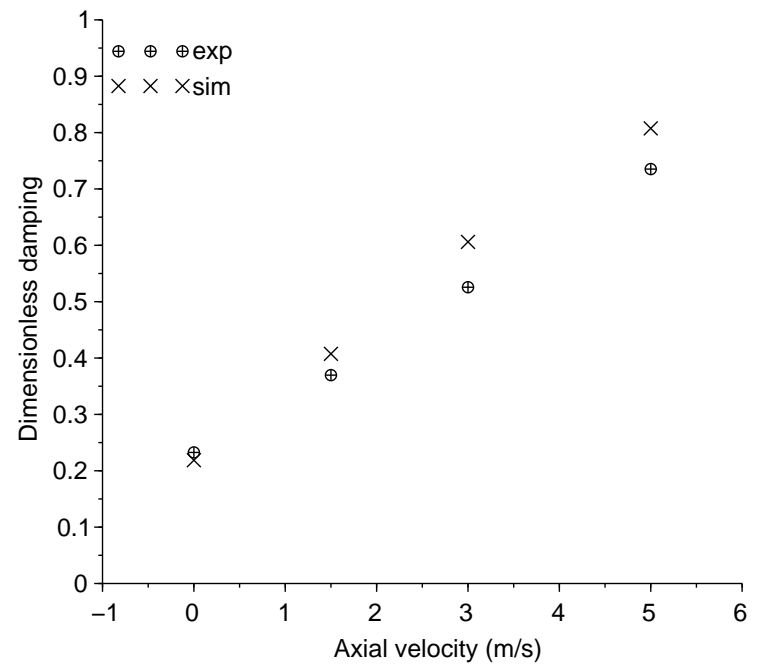

Fig. 16. Damping parameter.

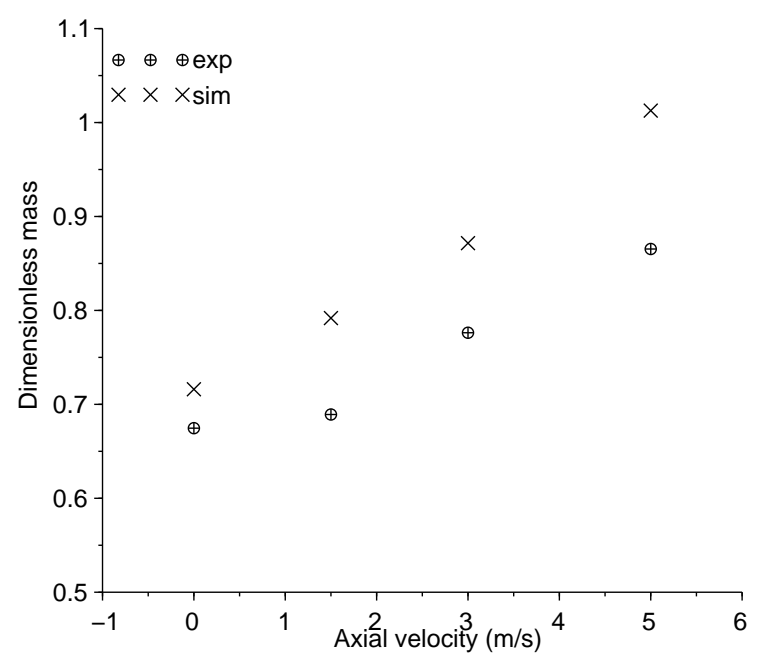

Fig. 17. Mass parameter. 


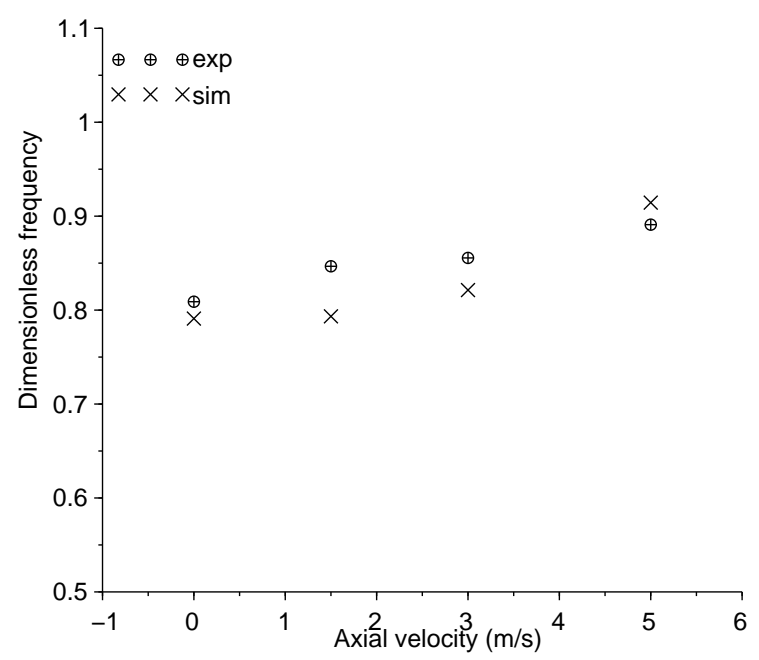

Fig. 18. Resonance frequency.

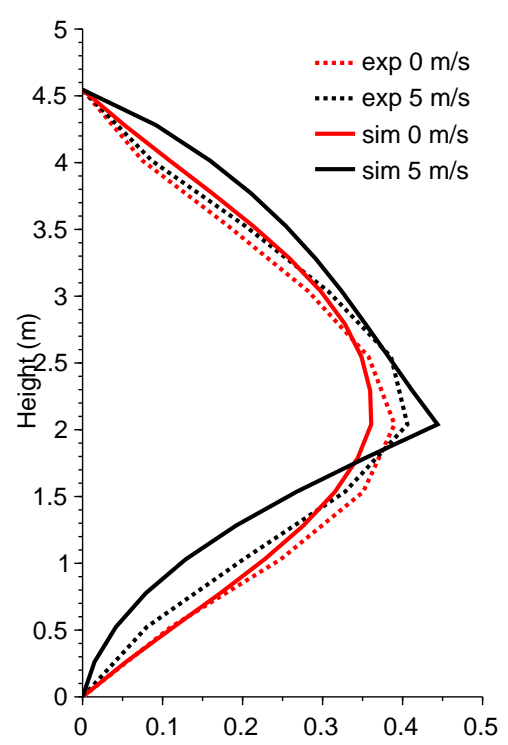

Fig. 19. Mode shape at resonance in still water and under axial flow. 